\title{
Capture, processing, and display of real-world 3D objects using digital holography
}

\author{
(Invited Paper)
}

\author{
Thomas J. Naughton $^{\triangle, *}$, Claas Falldorf ${ }^{\dagger}$, Levent Onural ${ }^{\ddagger}$, Pietro Ferraro ${ }^{\S}$, Christian Depeursinge ${ }^{\llbracket}$, \\ Sven Krueger ${ }^{\star}$, Yves Emery ${ }^{\diamond}$, Bryan M. Hennelly ${ }^{\triangleleft}$, and Małgorzata Kujawiñska ${ }^{\perp}$ \\ *University of Oulu, Oulu Southern Institute, 84100 Ylivieska, Finland. Email: firstname.lastname@oulu.fi \\ ${ }^{\dagger}$ BIAS - Bremer Institut für Angewandte Strahltechnik Gmbh, Bremen, Germany \\ ${ }_{\ddagger}^{\ddagger}$ Electrical and Electronics Engineering Department, Bilkent University, Ankara, Turkey \\ §Istituto Nazionale di Ottica Applicata, Consiglio Nazionale delle Ricerche, Napoli, Italy \\ 『Advanced Photonics Laboratory, École Polytechnique Fédérale de Lausanne, Switzerland \\ ${ }^{\star}$ HOLOEYE Photonics AG, 12489 Berlin, Germany \\ ${ }^{\diamond}$ Lyncée Tec Inc, 1015 Lausanne, Switzerland \\ ${ }^{\triangle}$ Department of Computer Science, National University of Ireland Maynooth, Ireland \\ ${ }^{\perp}$ Faculty of Mechatronics/Institute of Micromechanics and Photonics, Warsaw University of Technology, Poland
}

\begin{abstract}
Digital holography for 3D and 4D real-world objects' capture, processing, and display" (acronym "Real 3D") is a research project funded under the Information and Communication Technologies theme of the European Commission's Seventh Framework Programme, and brings together nine participants from academia and industry (see www.digitalholography.eu). This three-year project marks the beginning a long-term effort to facilitate the entry of a new technology (digital holography) into the three-dimensional capture and display markets. Its progress at the end of year 2 is summarised.
\end{abstract}

\section{INTRODUCTION}

Current and newly-developed 3D displays have the disadvantage of requiring special eyewear, limit the number of simultaneous viewers, discard completely certain depth cues (such as blurring) thus causing fatigue, or else encode only a small number of distinct different views. It can be argued that there is only one known technology that can capture a full 3D scene in a single shot, including phase information, and re-project that light field perfectly thus overcoming all of the above disadvantages: holography. All other techniques are only $3 \mathrm{D}$ under a whole host of conditions.

Unfortunately, conventional holograms are not dynamic. By replacing the conventional holographic plate with a digital camera and an optoelectronic 2D screen, we can capture and display holographic video. However, the full implications of bringing a digital version of holography into the world of 3D video acquisition and 3D display, or how effective it would be, are as yet unknown. The full 3D information encoded in digital holograms has not yet been exploited.

The project "Digital holography for 3D and 4D real-world objects' capture, processing, and display" is funded under the Information and Communication Technologies theme of the European Commission's Seventh Framework Programme, and brings together nine participants from academia and industry. This three-year project marks the beginning a long-term effort to facilitate the entry of a new technology (digital holography) into the three-dimensional capture and display markets. Within the three years, we will work towards eliminating current obstacles to achieving the world's first fully functional 3D video capture and display paradigm for unrestricted viewing of real-world objects that employs all real 3D principles, hence our acronym "Real 3D." The primary outputs are:

Output 1. A 3D holographic acquisition system based on digital camera technology, with at least 8 million pixels of side length no greater than $9 \mu \mathrm{m}$, arranged nonuniformly in a circular configuration around a space of diameter $10 \mathrm{~cm}$ that will be capable of holding a real-world 3D scene. We may even exceed these targets depending on how sensor technology improves over this year. The acquisition system will be capable of recording holographic video of the 3D scene.

Output 2. A 3D holographic display system based on liquid crystal on silicon (LCOS) technology, with at least 3 million pixels of side length no greater than $12 \mu \mathrm{m}$, arranged nonuniformly in a circular configuration of diameter of at least $10 \mathrm{~cm}$. Again, we may even exceed these targets depending on how LCOS technology improves over this year. The reconstruction system will be capable of displaying holographic video of the 3D scene.

Output 3. The signal/image/information processing theories, techniques, and tools required for the processing, analysis, and synthesis of the data from capture to display, including adapting the data captured for display on alternative configurations and on conventional 3D displays.

Output 4. Reports containing the hard scientific data, in terms of functionality, performance, resolution, restrictions, data quality, and visual perception, that would be required by a company to take our proof-of-concept outputs and develop the next stage in the commercialisation of this 3D technology. In addition, reports on the theories, techniques, and tools that 
enable this technology.

Functional models of four digital holographic 3D capture, processing, and display scenarios will be developed to target the work, encompassing (Scenario 1) the full $360^{\circ}$ of perspectives of reflective macroscopic 3D scenes, (Scenario 2) microscopic reflective 3D scenes, (Scenario 3) transmissive or partially transmissive microscopic 3D scenes, and (Scenario 4) capture of $3 \mathrm{D}$ scenes at infra-red wavelengths.

\section{DESCRIPTION OF THE WORK PERFORMED SINCE THE BEGINNING OF THE PROJECT}

Since the beginning of the project, a large portion of the work has been assigned towards analysis of the capabilities and fundamental limits of the technology and principles. In order to guide the project, a significant analysis of the capabilities, tools, and techniques of digital holography in the context of 3D information representation has been undertaken. A taxonomy of super-2D data representations has been devised. An analysis of all of the properties and potential future functionality of a digital hologram file format has been undertaken. A study of the state-of-the-art in digital holographic and non-digital holographic 3D imaging techniques has commenced with the view to finding synthesis opportunities, as has a survey of the state-of-the-art in dynamic holographic displays.

Since the beginning of the project most of the practical work has been performed on the capture side of the captureprocessing-display chain. The $360^{\circ}$ primary hologram capture arrangement is in its late design stages, and a portion of the circle is close to being implemented. Techniques to overcome flaws in existing hologram designs (keyhole problem, twin image presence) are under development ranging from optical solutions, solutions involving digital image processing, and solutions requiring the capture of multiple different holograms of the same scene. Experiments with Fresnel holography, lensless Fourier holography, rainbow digital holography, phaseshifting digital holography, and infrared holography have been performed. Separately, work has gone into non-real-time optical arrangements for capturing $360^{\circ}$ hologram data of objects and several hologram videos of rotated microscopic and macroscopic objects have been captured.

In terms of processing of holograms, besides solutions to the keyhole and twin problems, a study has commenced on the extension of state-of-the-art single-frame hologram data compression techniques for hologram video. Reconstruction on tilted planes has also been studied as an aid to more effectively extract information about 3D objects encoded in digital holograms. Extended focus imaging in digital holography overcomes the naturally small depth of field in reconstructions.

In terms of display, a general description of the data management process has been completed that connects capture hardware and techniques, processing, and optoelectronic reconstruction. Work on optoelectronic display of digital holograms, including colour holograms, has commenced and the first results have been obtained. Modification and display of digital holograms using conventional display technology has commenced, and a head-tracking monocular display that aims to give the illusion of a 3D object has been developed. A visual perception study is being planned to evaluate its success.

Significant effort has gone into dissemination activities, including many journal articles, invited and contributed conference papers, general-public presentations, newspaper interviews, international technical magazines, webpage with paper reprints and quarterly news updates, and three patent applications in various stages of preparation. Close interactions have been formed with several other EC-funded projects and meaningful collaborations are in their early stages. This impact can be illustrated by the fact that multinational companies (from inside and outside Europe) have been sufficiently interested in the Consortium's work to have requested to join the Consortium to obtain advanced access to technical results.

\section{DESCRIPTION OF MAIN RESULTS ACHIEVED SO FAR}

The main results achieved so far are:

- Complete state-of-the-art reports on 3D acquisition and $3 \mathrm{D}$ display techniques, what kind of 3D information is present in digital holograms, how that information can be made accessible, and analysis of compatibilities between the data from different kinds of sensing techniques.

- The project has aligned itself with four specific scenarios to focus research efforts (see end of Sect. I for list).

- In Scenario 1, a compact hologram capture device has been developed, hologram requirements have been specified to maximise viewer impact, a comprehensive report has been developed on numerical twin and speckle reduction techniques with several examples implemented, the best procedures for hologram processing have been identified, hologram video has been compressed, the display side components have been fully specified and evaluated experimentally, and visual perception experiments have been designed.

- In Scenario 2, holograms of microscopic objects have been captured with dual wavelengths, the data has been processed to admit extended focus images and reconstructions on tilted planes, the data has been conditioned for conventional stereo displays and to allow efficient manipulation of 3D properties of scenes, and visual perception experiments have been conducted.

- In Scenario 3, the two-axis rotation setup has been finalised and data has been captured of transmissive biological specimens. On the processing side, a tomographic reconstruction algorithm has been finalised to accurately obtain volumetric data. For display, multi-color holographic reconstructions from phase-only SLMs have been obtained using LED illumination.

- In Scenario 4, holograms have been acquired in the infrared and promising novel applications have been demonstrated such as capturing objects of relatively large dimensions and silicon defect analysis. Procedures are available to optoelectronically display the resulting data on versatile relatively-compact setups and on widerviewing-angle setups using multiple displays. 


\section{EXPECTED FINAL TECHNICAL RESULTS AND THEIR POTENTIAL IMPACT AND USE}

The project work is divided into nine work packages (WPs), the first of which concerns administrative management and technical coordination. WP 2 "Strategies for 3D information extraction" will allow the public (general and scientific) to share a common knowledge on the state of the art of the various features of holographic technology as well as to share a common language and terminology for definitions and concepts. The resulting report is almost exclusively public access on the project website, and represents the scientific/technical basis for discussions for later WPs in Real 3D and wider afield. It will allow industrial entities new to digital holography to immediately gain a practical overview of the capabilities of digital holography, written by the experts in the field, and extremely timely. The digital hologram file format will define a standard enabling interoperability between groups, and products, lowering costs and ensuring stability.

At the end of the project, WP3 "Hologram capture" will have resulted in strategies for capturing reflecting and transparent microscopic/macroscopic objects in different scenarios that will have been formulated and experimentally verified. A capture system capable of capturing wavefields reflected from a macroscopic object simultaneously from six directions will be in action. This experimental proof of concept will supply the necessary evidence to enthuse industry.

WP4 "Hologram pre-processing" is an enabling workpackage that will allow more efficient hardware implementations through the invention of novel procedures for noise reduction and resolution enhancement, thus making high resolution digital holography more economical.

By collecting full information on digital holographic and non-digital holographic techniques for 3D imaging and display, and by performing comparisons of their performances and evaluation of their compatibility or complementarities, WP5 "Compatibility with other technologies" aims at situating the currently maturing $3 \mathrm{D}$ digital holographic techniques in the context of existing technologies. From this work, industrialists and laboratories willing to develop real 3D imaging will have at their disposal a concrete basis and a comprehensive view of the ways digital holography can be introduced in the world of the existing $2 \mathrm{D}$ and $3 \mathrm{D}$ acquisition and display technologies, including helpful studies beyond visible wavelengths and software tools for data handling. This will more easily allow the transition of existing closely related industry to move into the digital holography field, and will promote the opportunity for novel fruitful marriages between digital holography technology and existing technologies. This analysis takes into account the emerging technologies that may be used in the future. WP5 will also provide coding and compression software to facilitate online networking applications and products.

The expected results of WP6 "Data management for numerical reconstruction" are to provide algorithms and tools allowing convenient interaction with $3 \mathrm{D}$ digital and optical hologram reconstructions. The different modalities will be totally transparent to the user who will be able to modify intuitively some parameters while immersed in the 3D reconstruction (point of view, reconstruction distance, and so on). These results will be essential if practical commercial products are envisaged, allowing ease of use and interactivity.

In WP7 "Data management for optoelectronic reconstruction" the procedures will be developed for enhanced holographic data preparation for flat displays (including multispectral data), and for multiple displays in a circular configuration thus laying the groundwork for future curved displays. By determining the most serious sources of error at reconstruction stage, numerical and experimental strategies will be developed to overcome or limit the influence of these errors on the final 3D reconstruction configuration. This will reduce the cost of eventual trials and provide important information for any digital hologram display manufacturing concerns.

Better scientific and technical understanding of dynamic holographic displays for holographic video, and advancements in such displays, will be one long-term influential output of WP8 "Novel displays." A laboratory proof of concept device consisting of many SLMs in a piecewise circular arrangement will be constructed. As 3D TV becomes more popular, we expect the positive results of this device will attract strong public attention and will further boost interest in 3D technologies. Each of the displays developed in this WP will have the potential to spark the initial interest of companies and venture capitalists by showcasing novel possibilities for the technology.

As well as annual reports, the outputs of WP9 "Exploitation and Dissemination" will include a report on the plans for exploitation and dissemination of the research results, and a report on how the project helped the environment for BIAS, HOLOEYE, and Lyncée Tec and SMEs in Europe in general in this specialised area. Recommendations will be made for future research and further development of digital holography in the context of 3D displays. An industry-focused report will include the possibilities and barriers for commercialisation of the Real 3D technology (sensing, processing, and display). This report targeted at industry (SMEs and large companies) will argue that the time is now right to start investing in digital holography. A report will describe the wider societal implications of the work. While the technical results and industrial impact are straightforward to formulate, the socioeconomic impact is less obvious but no less important. It is envisaged that 3D displays will increase productivity, may save lives in a medical scenario (such as keyhole surgery, or better diagnosis through holographic microscopy), and may have wider societal impact in terms of improved education and personal communication experiences.

This research received funding from the European Community's Seventh Framework Programme FP7/2007-2013 under grant agreement no. 216105 .

\section{REFERENCES}

[1] Please go to http://www.digitalholography.eu/publications.html for reprints of all of the research results described here. 